\title{
Two distinct neuronal populations in the rat parafascicular nucleus oppositely encode the engagement in stimulus-driven reward-seeking
}

3 Mehdi Sicre $^{1}$, Julie Meffre ${ }^{1}$ and Frederic Ambroggi ${ }^{1}$

4 'Aix-Marseille Université, CNRS, Laboratoire de Neurosciences Cognitives, UMR 7291, Team

5 Motivation, Marseille, France.

6 Corresponding author: Frederic Ambroggi

7 Laboratoire de Neurosciences Cognitives

8 UMR CNRS 7291 - Aix-Marseille Université

9 3, Place Victor Hugo, Case C

1013331 Marseille Cedex 3, France

11 Office: +33(0)4 13550937

12 Fax: +33(4)13550958

13 Email: frederic.ambroggi@univ-amu.fr

\section{ABSTRACT}

The thalamus is a phylogenetically well-preserved structure. Known to densely contact cortical regions, its role in the transmission of sensory information to the striatal complex has been widely reconsidered in recent years. The parafascicular of the thalamus (Pf) has been implicated the orientation of attention towards salient sensory stimuli. In a stimulus-driven reward seeking task, we sought to characterize the electrophysiological activity of Pf neurons in rats. We observed a predominance of excitatory responses over inhibitory responses for all events of the task. Neurons responded more strongly to the stimulus compared to leverpressing and collecting reward, confirming the strong involvement of the Pf in sensory information processing. The use of long sessions allowed us to compare neuronal responses to stimuli when the animal engaged in action or when it did not. We distinguished two populations of neurons responding in an opposite way: MOTIV+ neurons responded more intensively to stimuli followed by a behavioral response than those that did not. Conversely, MOTIV- neurons responded more strongly when the stimulus was ignored by the animal. In addition, MOTIV- neurons excitations appeared at a shorter latency after the stimulus than MOTIV+ neurons. Through this encoding, Pf could perform an early selection of environmental stimuli transmitted to the striatum according to motivational level. 


\section{ABBREVIATIONS}

32 CIN: Cholinergic interneurons

$33 \mathrm{CM}$ : Center médian nucleus of the thalamus

34 FR: Fixed-ratio

35 LLF: long-latency facilitation

36 MSN: Medium spiny neurons

37 NAc: Nucleus accumbens

38 Pf: Parafascicular nucleus of the thalamus

39 PSTH: Peri-stimulus time histograms

40 SLF: short-latency facilitation

\section{HIGHLIGHTS}

- Pf neurons respond to reward-predicting stimuli and reward-related actions

- MOTIV+ Pf neurons were more active to stimuli evoking reward-seeking

- MOTIV- Pf neurons were more active to stimuli ignored by the animal

- Stimuli-evoked excitations latencies were shorter in MOTIV- than MOTIV+ neurons

\section{KEYWORDS}

47 Parafascicular nucleus, thalamus, attention, motivation, incentive stimuli, reward-seeking, 


\section{INTRODUCTION}

The historic view of the thalamus as an ensemble of nuclei relaying sensory information to the cortex (Ariens-Kappers et al., 1936) has largely been reconsidered. The thalamus is a phylogenetically ancient structure that evolved before the expansion of the neocortex (Butler, 1994). Thus, if thalamic nuclei unequivocally provides essential information to the cerebral cortex through theirs projections, it is not surprising that they also intensively connects subcortical regions (James et al., 2011; Rikhye et al., 2018). In particular, the associative thalamic nuclei from the midline and intralaminar group send strong projections to the striatal complex (Van der Werf et al., 2002). This structure is considered the entry station of the basal ganglia, a subcortical network participating in the selection and the control of voluntary actions (Redgrave et al., 1999; Hikosaka et al., 2014; Sirigu and Duhamel, 2016). This pattern of connectivity has challenged a key element of the classical model of the basal ganglia stating that the input signal to the striatum originates from the cerebral cortex (Alexander, 1986). Some authors even radically proposed that the thalamus, rather than the cortex, may be the dominant source of sensory information to the basal ganglia (McHaffie et al., 2005; Redgrave and Gurney, 2006; Redgrave et al., 2011). This appreciation of thalamic afferences to the striatum has largely incentivized their investigation at the cellular and network level (Ding et al., 2010; English et al., 2012; Doig et al., 2014; Mandelbaum et al., 2019) and several studies have now begun to address their functional implications at the behavioral level (Matsumoto et al., 2001; Hamlin et al., 2009; Bradfield et al., 2013; Do-Monte et al., 2017; Díaz-Hernández et al., 2018; Meffre et al., 2019).

In particular, the primate center médian (CM)/parafascicular nucleus (Pf) complex from the caudal intralaminar nuclei has attracted a lot of attention. The $\mathrm{CM}$ is not present in the rodent brain but it has been largely documented that the primate CM/Pf complex is equivalent to the rodent Pf (Jones and Leavitt, 1974; Smith et al., 2004, 2011). An extensive topographic projection follows a medio-lateral axis in the Pf to a ventromedial-dorsolateral axis in the striatal complex in both rats (Berendse and Groenewegen, 1990, 1991; Van der Werf et al., 2002) and mice (Mandelbaum et al., 2019). The medial Pf projects to the ventral region of the striatum (i.e. nucleus accumbens, NAc) while the lateral Pf projects to the dorsolateral striatum. A similar topography has also been described in primates (Jones and Leavitt, 1974; Groenewegen and Berendse, 1994; Smith et al., 2004). 
81 Electrophysiological recordings have shown that primate CM/Pf neurons are rapidly excited by stimuli from a variety of sensory modalities (auditory, visual and somatosensory). These activations depend on the temporal unpredictability of stimuli and their association with rewards (Matsumoto et al., 2001), suggesting that the CM/Pf is involved in attentional orientating toward salient sensory stimuli (Minamimoto and Kimura, 2002; Kimura et al., 2004). Furthermore, it has been reported that CM/Pf neurons are more excited by stimuli predicting a small than a large reward (Minamimoto et al., 2005) and that the magnitude of these excitations inversely correlate with reaction times (Minamimoto et al., 2014).

Thus, the CM/Pf could provide important information to the striatum to adequately control the engagement in action in response to temporally unexpected stimuli predictive of rewards. To our knowledge, the response of Pf neurons to reward-predictive stimuli has not been investigated in rodents. The aim of this study was to fill this gap by recording single neuron activity in the Pf in a task where rats had the opportunity to engage in reward-seeking by performing an instrumental action in response to a predictive stimulus. We used long sessions to compare Pf neuronal responses to stimuli when rats decided to engage in reward-seeking and when they did not. We found a population of neurons that responded more intensively to reward-predicting stimuli the animals responded to compared to those they ignored. A second population, excited to stimuli at shorter latency, had an opposite pattern of activity. We propose that these two populations may respectively promote and refrain the engagement in action through their connections to the NAc.

\section{EXPERIMENTAL PROCEDURES}

\section{Subjects}

Experiments were conducted on male Long-Evans rats (Charles Rivers, France) weighing 300g on arrival. Rats were immediately housed individually on a $12 \mathrm{~h}$ light/dark cycle. Experiments were conducted during the light phase. After one week of habituation, rats were placed under food restriction; food rations were adjusted daily to maintain the body weight at $\sim 90 \%$ of their free-feeding body weight. All experiments were performed in accordance with the guidelines on animal care and use of the European guidelines (European Community Council Directive, 2010/63/UE) and National guidelines. 
111 All experiments were conducted in operant chambers containing two house lights, a tone 112 speaker, a retractable lever and a reward receptacle located on one wall of the chamber (Med 113 Associates, Vermont, USA). Liquid sucrose (10\%) was delivered as a reward in the receptacle 114 by a syringe pump. During the first 2 days, rats were trained to obtain $50 \mu$ l of sucrose by spontaneously entering into the reward receptacle. When 300 rewards per hour were obtained, rats were run on a fixed-ratio 1 (FR1) schedule with a 10 s-time-out: the lever was constantly extended in the chamber and a lever-press triggered the delivery of $50 \mu$ l of sucrose into the receptacle. When rats reached the criterion $>100$ lever-presses per hour, they were advanced to a stimulus-driven reward-seeking FR1 task.

Stimulus-driven reward-seeking task

121 Rats were run daily on the task for 3 hours. The stimulus was composed of the extension of 122 the lever associated with a $300 \mathrm{~ms}$ white noise $(85 \mathrm{~dB})$. When the stimulus was presented, 123 rats had $10 \mathrm{~s}$ to press the lever to obtain the reward. Each trial was followed by a variable 124 interval schedule averaging $45 \mathrm{~s}$ (from 30 to $60 \mathrm{~s}$ ). If the rats did not respond on the lever 125 within $10 \mathrm{~s}$, the lever retracted and the intertrial interval was re-initialized. Surgery was 126 performed when rats reached the criterion of $>80 \%$ responses during the first hour of the 127 session.

128

129

\section{Surgery}

Rats were anesthetized with isoflurane (5\%, Tem Sega, France) and placed in a stereotaxic apparatus (Kopf Instruments, California, USA). Anesthesia level was then adjusted with 0.5-2 $\%$ isoflurane during the maintenance phase. Before skin incision, a subcutaneous injection of lidocaine $(1 \mathrm{mg} / \mathrm{ml}$, Lurocaine MedVet,) was performed. Bundles of 8 electrodes were attached to custom-made-microdrive devices (du Hoffmann et al., 2011) that allowed to lower the electrode bundles by $80 \mu \mathrm{m}$ increments. Electrodes were implanted in the Pf bilaterally for 5 animals and unilaterally in 2 animals (one in the left and the other in the right hemisphere) at the following stereotaxic coordinates: AP: $-4.1, \mathrm{ML}:+/-1, \mathrm{DV}:-5.4 \mathrm{~mm}$ relative to the Bregma. The microdrive and the connector were secured to the skull with bone screws, adhesive cement (C\&B Metabond, Phymep, France) and dental acrylic (Phymep, France). After surgery, a prophylactic analgesic treatment (Buprenorphine, $0.05 \mathrm{mg} / \mathrm{kg}$, Vetergesic, France) 
140 was administered, and rats were given at least 7 days of recovery with ad libitum access to

141 food. After recovery, rats were placed in food restriction and re-trained until reaching 142 previous criteria.

\section{Electrophysiology}

\section{Recording procedure}

145 Electrophysiological recordings were conducted as described previously (Sicre et al., 2020) 146 during the entire 3 hour-long sessions. Animals were connected to the electrophysiological 147 acquisition system (SpikeGadget LLC, California, USA). The 32-channel headstage streamed 148 data at $30 \mathrm{kHz}$ per channel and was connected to a low-torque HDMI commutator that allowed 149 the animals to be free of their movement in the chamber. Between sessions, electrode 150 bundles were lowered by 80 or $160 \mu \mathrm{m}$ to record news set of neurons. Unfiltered data were 151 transferred from the data acquisition main control unit to a data acquisition computer where 152 it was visualized and saved. Digitally-filtered data $(0.2-6 \mathrm{kHz})$ was used for spike sorting.

\section{Spike sorting}

154 Recorded data were analyzed offline with OfflineSorter (Plexon Inc, Texas, USA) to isolate 155 spikes from single neurons with principal component analysis. Inter-spike interval 156 distributions, cross-correlograms and auto-correlograms were used to ensure that the activity 157 of single neurons was isolated. Only well isolated waveforms with characteristics that were 158 constant over the entire recording session were included in this study. Sorted units were 159 exported to NeuroExplorer 4.135 (Nex Technologies, Colorado, USA) and Matlab R2018b 160 (MatWorks Inc, Massachusetts, USA) for further analysis.

\section{Electrophysiological analyses}

\section{Waveform Analysis}

163 In our data set, 290 neurons showed waveforms with a negative followed by a positive 164 deflection. The remaining 95 neurons displayed the opposite pattern. The spike width was 165 assessed by the time elapsed between the first and second extremum independently from the 166 sign of the first and second deflections. The spike width of 14 neurons could not be 
167 determined due to technical issues and we then excluded from the analyses involving this parameter.

170 Peri-stimulus time histograms (PSTHs) were constructed with smoothed (lowess method, 171 span=4) $20 \mathrm{~ms}$ - and $2 \mathrm{~ms}$-time-bins. PSTHs constructed around the behavioral events (stimulus 172 presentation, lever-press and reward collection) were used to detect excitations and 173 inhibitions and the time at which they occurred. The $10 \mathrm{~s}$ period before the presentation of 174 the stimulus was used as a baseline period. Excitation and inhibition to each event was 175 determined by the presence of at least 3 consecutive bins above the $99 \%$ (for excitations) or 176 below the $1 \%$ (for inhibitions) confidence interval of the baseline during the analysis windows 177 (0 to $250 \mathrm{~ms}$ after the stimulus, -1000 to $500 \mathrm{~ms}$ around lever-presses and reward delivery). 178 Onset was determined by the time of the first of 5 consecutive bins falling outside the 179 confidence interval. The offset was determined in analogy, by searching the first of 10 180 consecutive bins within the confidence interval.

\section{Deconvolution}

182 To isolate the activity of temporally close events, we used a deconvolution method as 183 described previously (Ghazizadeh et al., 2010; Ambroggi et al., 2011). Briefly, the model 184 assumes that the total firing rate of a neuron in each trial is equal to the linear sum of the 185 contributions of each event-related firing, delayed by the event latencies in that trial. Here, 186 we deconvolved single event responses for each neuron using the optimal number of 187 iterations that had a cross validation error lower than the PSTHs.

Data normalization and plotting

189 Color-coded maps and average PSTHs across neurons were constructed with 20ms- and 2ms190 bins. Prior to averaging, the firing rate of each neuron during each bin was z-score191 transformed: $\left(F_{i}-F_{m e a n}\right) / F_{s d}$ where $F_{i}$ is the firing rate of the $i^{\text {th }}$ bin of the PSTH, and $F_{\text {mean }}$ and $192 \mathrm{~F}_{\mathrm{sd}}$ are, respectively, the mean and the SD of the firing rate during the 10 s preceding stimulus 193 onset. 
194

\section{Statistical analyses}

We sought to compared the trials to which the animal engaged in reward-seeking with those it did not. Thus, we selected the sessions containing at least 20 trials of each type to conduct reliable analysis of the neuronal data. For behavioral analyses, the primary dependent variables were the percentage of responding to the stimulus and the latency to lever-press after the presentation of the stimulus.

For electrophysiological data, the primary dependent variables were the baseline firing rate, the maximal frequency reached (measured by averaging the top 5\% of instantaneous frequencies during the baseline period), the coefficient of variation (measured during the baseline period), the spike width, the proportion of responsive neurons, the mean z-score normalized firing (0-100 ms post-stimulus and -500 to $1000 \mathrm{~ms}$ around lever-presses and reward collection), the onset latency and the durations of the responses. These variables were analyzed with paired, unpaired t-tests or ANOVAs. When appropriate, a Bonferroni test was used to conduct post-hoc comparisons. Proportions were analyzed with $\chi^{2}$ tests and distributions with Kolmogorov-Smirnov tests. All results were considered significant at $p<$ 0.05 .

\section{Histology}

Animals were deeply anesthetized with a solution of pentobarbital (Euthasol, $140 \mathrm{mg} / \mathrm{kg}$ ). A local anesthesia was performed on the thorax in the incision site (Lurocaine $10 \mathrm{mg} / \mathrm{kg}$ ). Each electrodes site was labeled by passing a DC current of $20 \mu \mathrm{A}$ for $7 \mathrm{~s}$. Rats were perfused intracardially with phosphate buffered saline following by $10 \%$ formalin solution. Brains where removed, post-fixed in $10 \%$ formalin and placed in $30 \%$ sucrose for 3 days. Brains were sectioned at $40 \mu \mathrm{m}$ on a cryostat and slices were stained with cresyl violet. Reconstruction of the recording sites was made based on the final location of the electrodes. 


\section{RESULTS}

\section{Behavioral analysis}

220 Rats were run in a stimulus-driven FR1 task where a 300ms-auditory stimulus was presented

221 unexpectedly (in average every $45 \mathrm{~s}$ ) in conjunction with the extension of a lever lasting up to

$22210 \mathrm{~s}$. A lever-press triggered the immediate delivery of a $10 \%$ sucrose reward in an adjacent

223 receptacle and the retraction of the lever (Fig. 1A, B). We sought to analyze neuronal activity

224 when the rats attended to the stimulus and when they did not. In order to obtain a sufficient

225 number of trials of each type, we ran 3-hour long sessions. For all rats, the percentage of lever-

226 pressing in response to the reward-predictive stimulus and the latency were variable across

227 sessions (5 to 9 sessions per rat for 7 rats, total of 46 sessions Fig. 1C). Overall, the mean

228 percentage of responding was $60.9 \pm 2.8 \%$ with an average stimulus-lever-press latency of

$2292.64 \pm 0.14 \mathrm{~s}$.

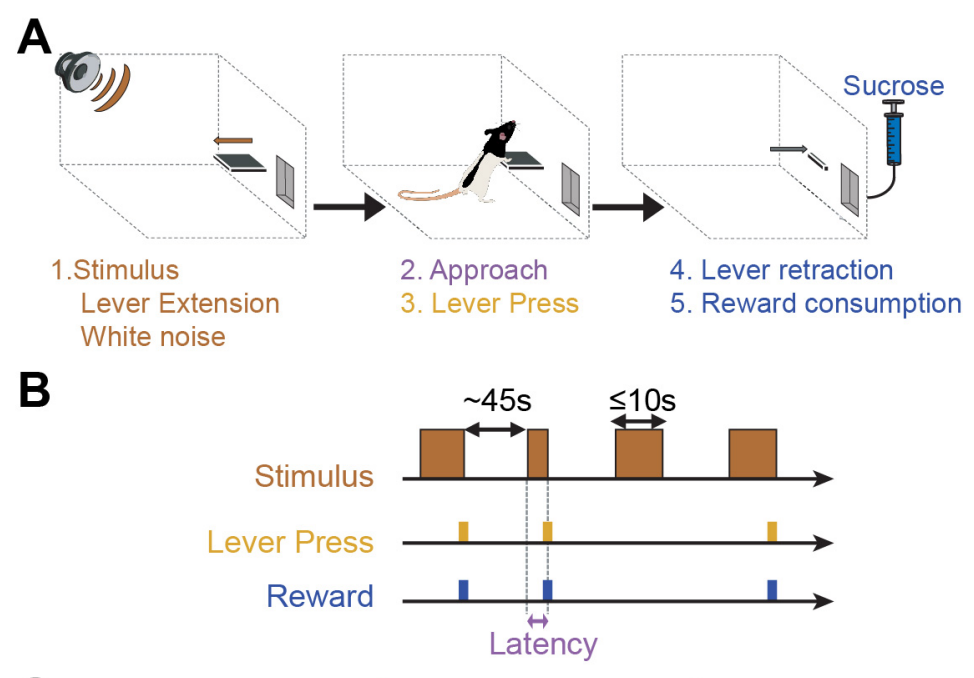

C

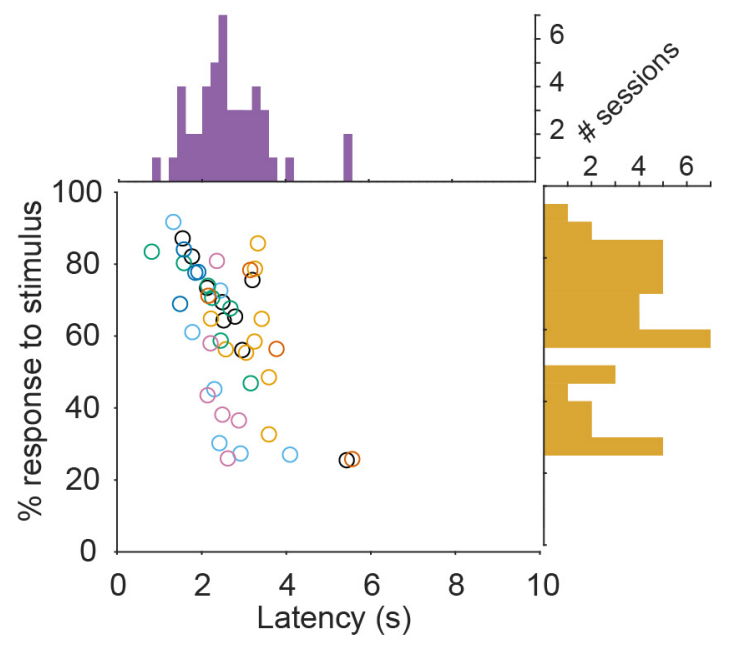

Fig.1. Behavioral performance in the stimulus-driven reward seeking task.

A. Task diagram showing the sequence of events in one trial. B. Temporal structure of the task. C. Individual percentage of responses and latencies for all sessions $(n=46)$. Animals $(n=7)$ are color-coded. Histograms represent the distributions of the percentage of responses (yellow) and latencies (purple). 


\section{Basic electrophysiological properties of Pf neurons}

231

We recorded single unit activity of 399 Pf neurons (see Fig. 2 for histological reconstruction of the electrode sites). We first conducted analyses of neuronal responses to the different events on the trials the animals responded to the stimuli by lever-pressing and collecting the rewards (Fig. 3).

We found that the majority of neurons were modulated by the different events with more excitations than inhibitions ( 254 and 88 neurons, respectively, $\chi^{2}=141, P<0.0001$ ). The excitations evoked by the stimulus were less frequent than those evoked by the lever-presses or the rewards $\left(155,229\right.$ and 228 neurons, respectively, $\chi^{2}=27.9 .0, P<0.0001$, Fig. $\left.3 \mathrm{~A}\right)$ and the majority of neurons were excited to more than one event (Fig. 3B). Inhibitory responses evoked by stimuli were also less frequent than those evoked by lever-presses or rewards (24, 82 and 78 neurons, respectively, $\chi^{2}=44.2, P<0.0001$, Fig. $\left.3 \mathrm{~A}\right)$. The inhibitory responses to the different events showed less overlap than excitatory responses $\left(\chi^{2}=37.2, P<0.0001\right.$, Fig. $\left.3 B\right)$. Across the population, we observed neurons with different waveform profiles and firing properties during the baseline period (i.e. 10 s preceding stimulus onset, Fig. $3 \mathrm{C}$ ). We analyzed separately the neurons excited or inhibited by task events. Event-excited neurons had an overall lower baseline firing rate (Kolmogorov-Smirnov test, $\mathrm{KS}=0.203, \mathrm{P}=0.007$, Fig. 3D) and a lower maximal frequency ( $K S=0.223, P=0.002$, Fig. $3 G$ ) than event-inhibited neurons. The distributions of spike widths and coefficients of variations were similar between excited and inhibited neurons ( $K S=0.125, P=0.255 ; \mathrm{KS}=0.130, \mathrm{P}=0.206$, respectively, Fig. $3 \mathrm{E}, \mathrm{H}$ ). When
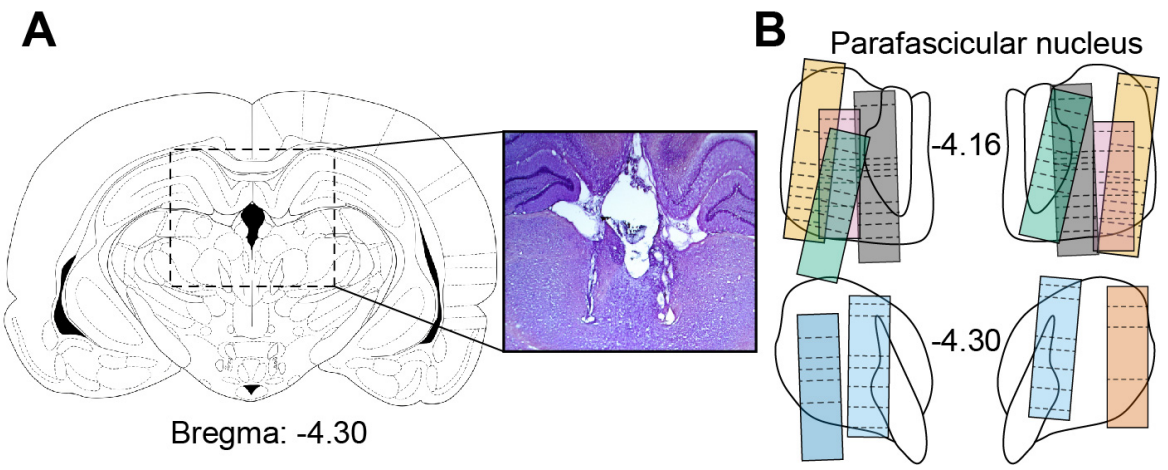

Fig.2. Histology and electrode path reconstruction.

A. Schematic diagram of a rat brain coronal section and representative photomicrograph of two electrode bundle tracks located in the Pf. B. Histological reconstruction of the recording sites in the Pf. Boxes represent the approximate extent of the electrode bundles in the 7 animals (color coded). Dotted line corresponds to the depth recording sessions. 
A

EXCITATIONS

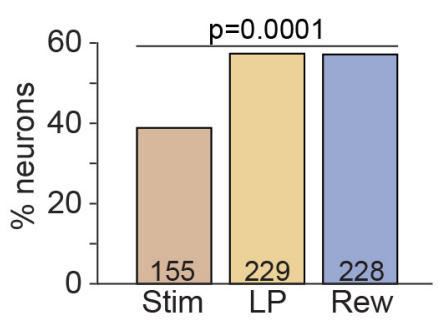

B

C

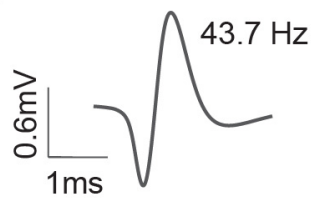

D

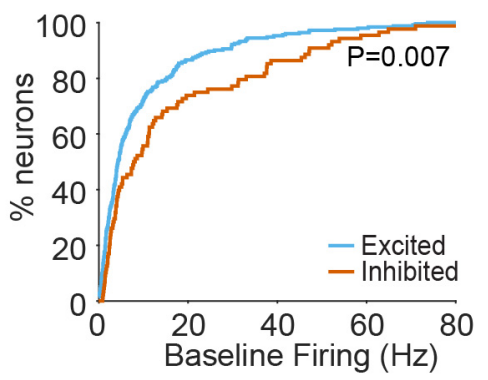

E

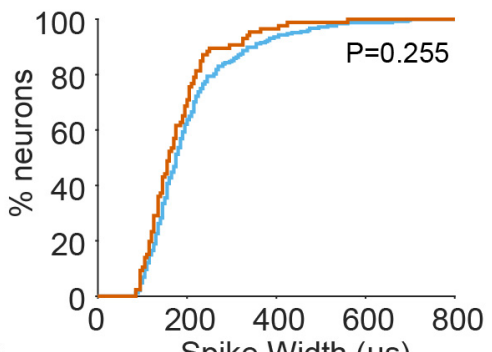

F

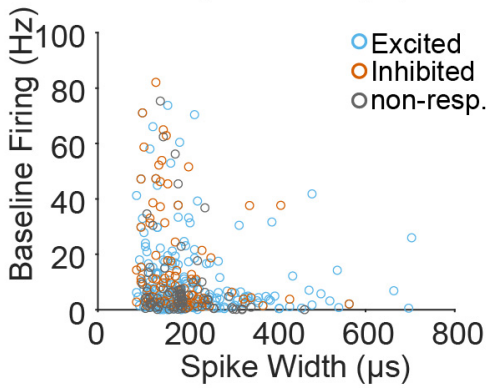

INHIBITIONS
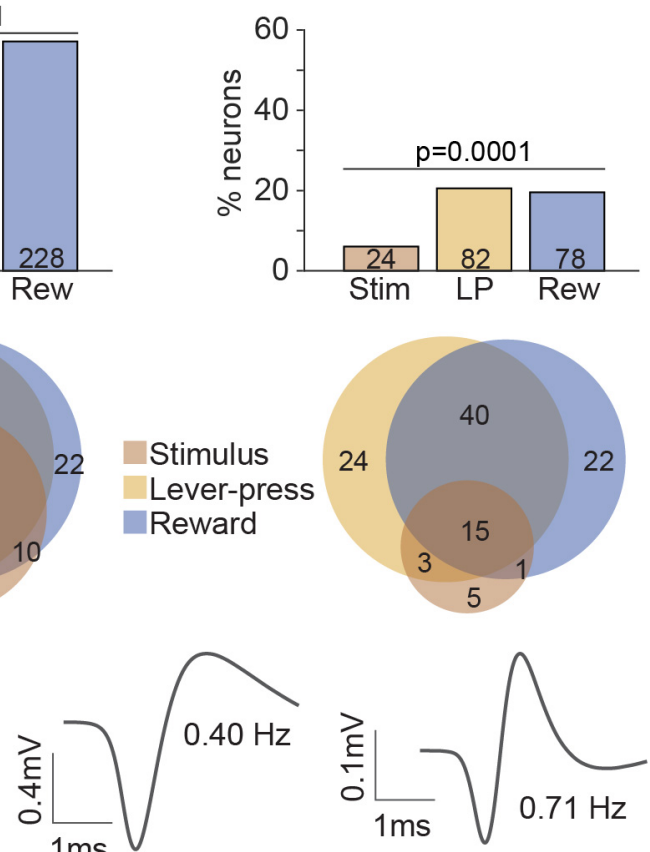

G
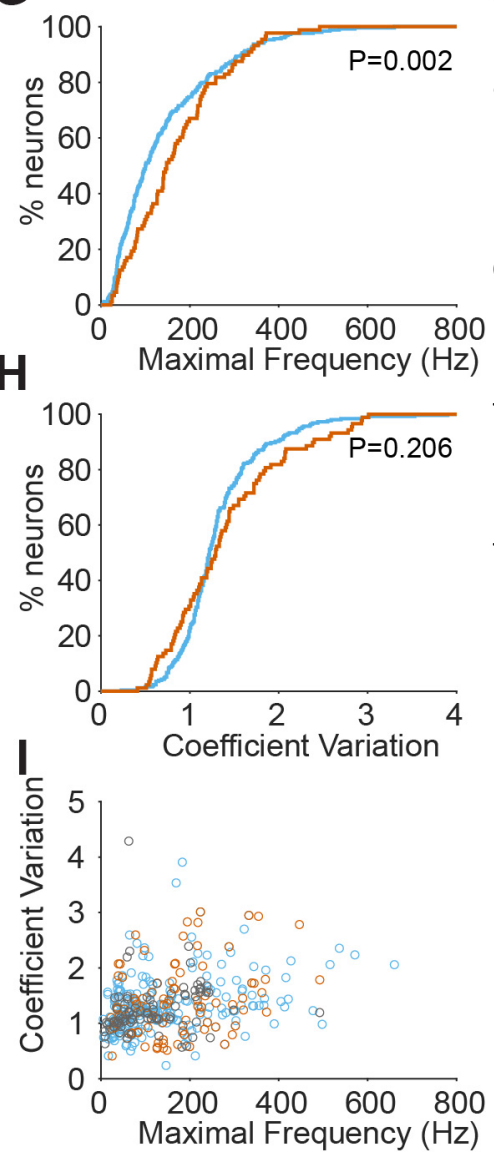

Fig.3. Electrophysiological characteristics of Pf neurons excited and inhibited by the different task events.

A. Percentage of neurons excited (left) and inhibited (right) by the different events (stimulus, lever-press, reward). B. Venn diagrams showing the proportion of neurons excited (left) and inhibited (right) by task events. C. Example of three representative waveforms recorded in the Pf with their baseline firing rate.

D. Cumulative percentage of baseline firing rate of taskexcited (blue) and taskinhibited (red) neurons.

E. Cumulative percentage of spike widths.

F. Baseline firing rate plotted against spike width for individual neurons (gray dots indicate non-responsive neurons).

G. Cumulative percentage of maximal frequencies

H. Cumulative percentage of the coefficients of variation.

I. Coefficient of variations plotted against the maximal frequencies for individual neurons. 
251 these variables were plotted against each other, event-excited and inhibited neurons strongly 252 overlapped and it did not allow to isolate excited from inhibited neurons based on these 253 different properties (Fig. 3F, I). We conducted similar analyses on excited and inhibited 254 neurons separately for each event and obtained similar results (data not shown). Taken 255 together, these results indicate that even if excited and inhibited neurons had different 256 properties, it was insufficient to separate them.

\section{Pf neuronal responses to task events}

258 We then analyzed the temporal dynamics and magnitudes of these different event-evoked responses that often lasted several seconds. But at the behavioral level, the stimulus, leverpresses and reward collections occur in close temporal proximity, often with smaller latencies than the duration of the neuronal responses. PSTHs constructed around each of these events can potentially be distorted by the presence of the other events that are correlated with each other in time. Thus, PSTHs do not allow one to accurately account for the neuronal responses to each individual event. To circumvent this issue, we used an iterative deconvolution method that takes advantage of the trial-to-trial variability in the temporal relationship between the different events to parse out the neuronal responses of each individual events from the PSTHs (Ghazizadeh et al., 2010). For each neuron, we deconvolved single-event responses by using the optimal number of iterations that had a cross-validation error lower than the PSTHs $(5.2+$ 0.2 iterations for the entire dataset, Fig. 4, 5). The analysis of raw PSTH aligned to the stimulus revealed that excitatory responses occurred at a stereotypical latency (58+/-4ms, Fig. 4A, C, D) with variable durations (1.57+/-0.17 s, Fig.

$2724 \mathrm{~A}, \mathrm{C}, \mathrm{E})$. The removal of the contribution of neighboring events by deconvolution had no 273 effect on the onset latency distribution of stimulus-evoked excitations (KS=0.147, P=0.09, Fig. 274 4D) but strongly decreased their durations (KS=0.278, $\mathrm{P}=3.4 \times 10^{-5}$, Fig. 4E).

275 With raw PSTH, most lever-press-evoked excitations emerged before the occurrence of the 276 event $(-0.830+/-0.05 \mathrm{~s})$ and lasted up to $4 \mathrm{~s}(1.26+/-0.11 \mathrm{~s})$. Reward-evoked excitations 277 occurred even earlier (-1.36+/-0.04 s) and lasted in average 1.18+/-0.10s (Fig. 4A, C, D). 278 Deconvolution significantly shifted lever-press- and reward-evoked excitations closer to the event (KS=0.226, $\mathrm{P}=4.3 \times 10^{-5}$ and $\mathrm{KS}=0.382, \mathrm{P}=3.0 \times 10^{-13}$, respectively, Fig. 4D) and considerably shortened their durations ( $\mathrm{KS}=0.25, \mathrm{P}=3.3 \times 10^{-6}$ and $\mathrm{KS}=0.26, \mathrm{P}=1.8 \times 10^{-6}$, respectively, Fig. $\left.4 \mathrm{E}\right)$. 
281 The analysis of the magnitude of evoked responses (Fig. 4F) revealed a strong influence of the event considered (2-way Repeated Measures ANOVA, Event effect, $F_{2,606}=123.6, P=9 \times 10^{-46}$ ) and of the deconvolution (Deconvolution effect, $F_{1,606}=355, P=1.1 \times 10^{-62}$ ) but no significant interaction (Event $x$ Deconvolution, $F_{2,602}=2.34, P=0.097$ ). Stimuli-evoked excitations were $\sim 3$ times significantly larger than those evoked by lever-presses and rewards (Bonferroni test on Event effect, $\mathrm{P}=2 \times 10^{-38}$ and $\mathrm{P}=8 \times 10^{-39}$, respectively) but lever-press- and reward-evoked excitations did not differ from each other.

Inhibitions analyzed on raw PSTHs shared many similarities with excitations, but deconvolution had less effect, suggesting that these responses were more temporally associated with the behavioral events (Fig. 5). Stimulus-evoked inhibitory responses occurred after $85+/-9 \mathrm{~ms}$ (non-significantly different from excitations, $\mathrm{KS}=0.264, \mathrm{P}=0.09$, Fig. 5A, B, D) and lasted $1.38+/-0.63 \mathrm{~s}$ (significantly shorter than excitations, $\mathrm{KS}=0.335, \mathrm{P}=0.0014$, Fig. $5 \mathrm{E}$ ). Deconvolution had no effect on these measures $(K S=0.175, P=0.85$ and $K S=0.25, P=0.44$, respectively, Fig. 5B-E).

296 Inhibitions to lever-presses and rewards preceded the events for most neurons $(-0.56+/-0.09$ $297 \mathrm{~S}$ and $-0.82+/-0.10$, respectively, Fig. 5A, C, D) and deconvolution had no effect (KS=0.18, $298 \mathrm{P}=0.18$ and $\mathrm{KS}=0.21, \mathrm{P}=0.14$, respectively, Fig. 5B, C, D). We observed a strong trend toward a 299 reduction of the duration of lever-press-evoked inhibitions by deconvolution (KS=0.22, $300 \mathrm{P}=0.053)$. The durations of reward-evoked inhibitions were reduced significantly $(\mathrm{KS}=0.312$, $301 \quad \mathrm{P}=0.006$, Fig. 5E).

302 The magnitude of inhibitory responses (Fig. 5F) depended on the event considered (2-way 303 Repeated Measures ANOVA, Event effect, $F_{2,181}=12.44, P=8.688 \times 10^{-6}$ ) and the deconvolution 304 (Deconvolution effect, $F_{1,181}=21.72, P=6.09 \times 10^{-6}$ ) but we found no significant interaction 305 (Event $x$ Deconvolution, $F_{2,181}=0.53, P=0.585$ ). Inhibitions to the stimulus were larger than 306 those to the lever-presses and rewards (Bonferroni test on Event effect, $\mathrm{P}=1.9 \times 10^{-5}$ and $307 \mathrm{P}=1.3 \times 10^{-5}$, respectively) but lever-press- and reward-evoked excitations did not differ from 308 each other.

309 Together, these results indicate that Pf neurons respond strongly to reward-predictive stimuli 310 but that are also modulated in anticipations of the actions of lever-pressing and the collection of the rewards. 
A

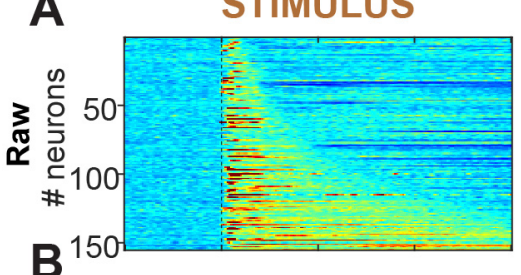

임

C

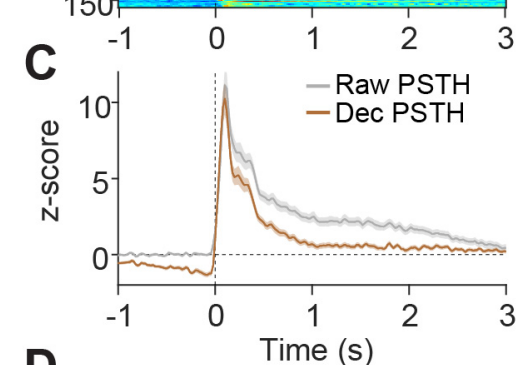

D
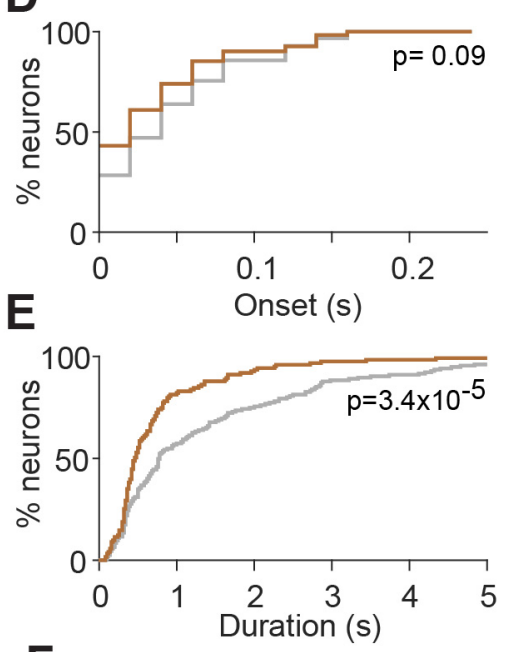

$\mathbf{F}$

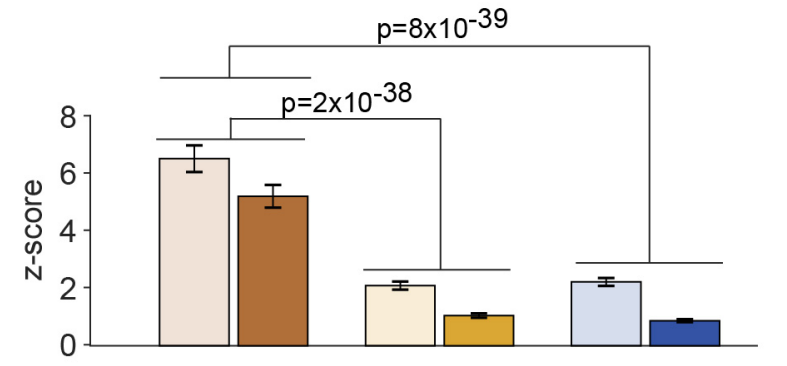

LEVER-PRESS
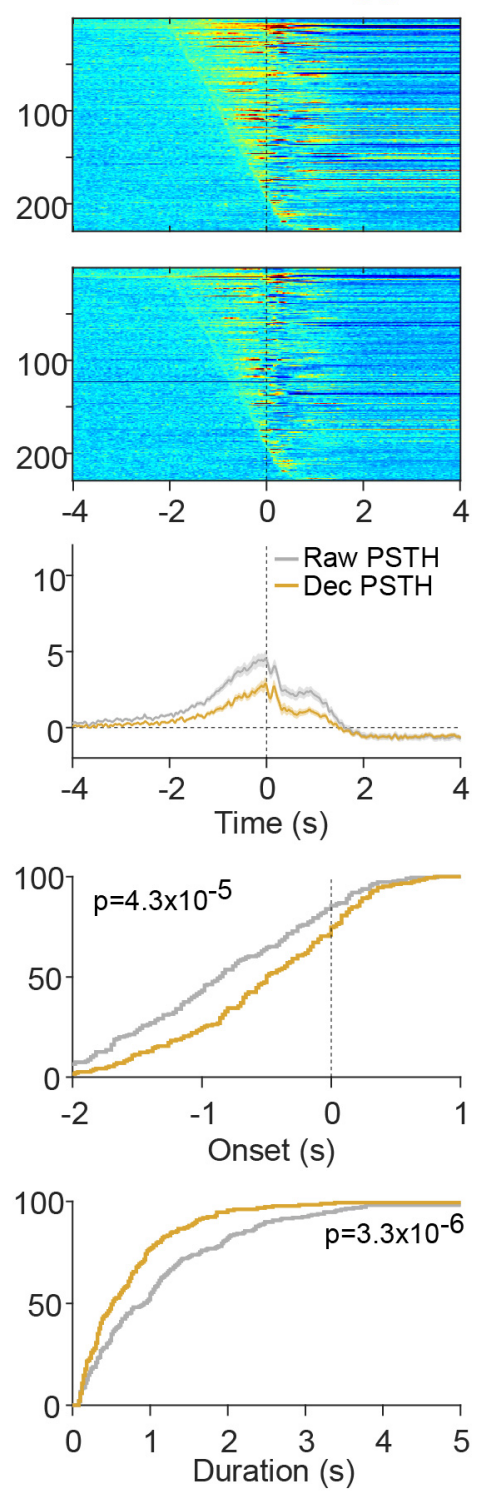
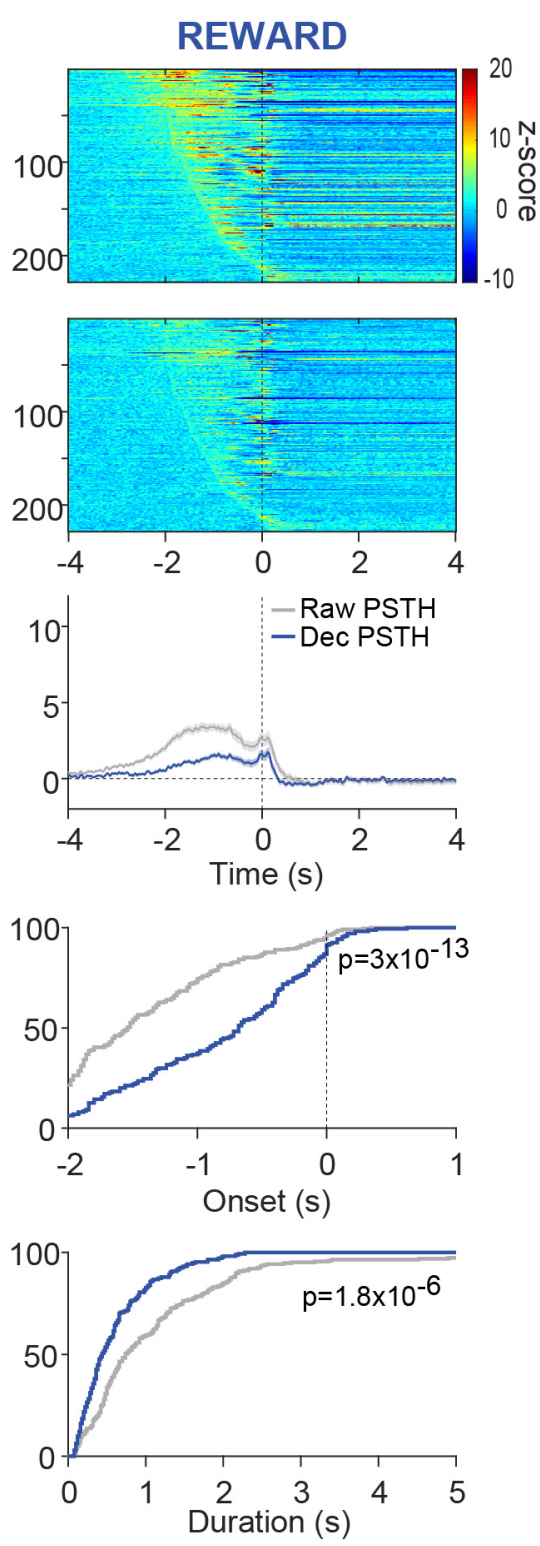

Fig.4. Pf neuronal excitations to task events.

A. Heat-maps represent color-coded PSTHs showing neurons excited to the stimulus (left), lever-press (middle) and reward delivery (right). Each row represents the PSTH an individual neuron aligned to the event considered. Data are plotted with smoothed $20 \mathrm{~ms}$-time-bins and neurons are sorted by excitation durations for stimuli responses and onset latencies for lever-press and reward responses. B. Heat-maps represent deconvolved PSTH of the same neurons represented in A. C. Average responses for the neurons shown in A and B. The gray traces correspond to raw PSTHs. Brown, orange and blue traces correspond to the deconvolved PSTHs to the stimulus, lever-press and reward delivery, respectively. D. Cumulative percentage of excitation onset latencies for raw and deconvolved responses. E. Cumulative percentage of excitations durations. F. Average z-scores of excitations. 

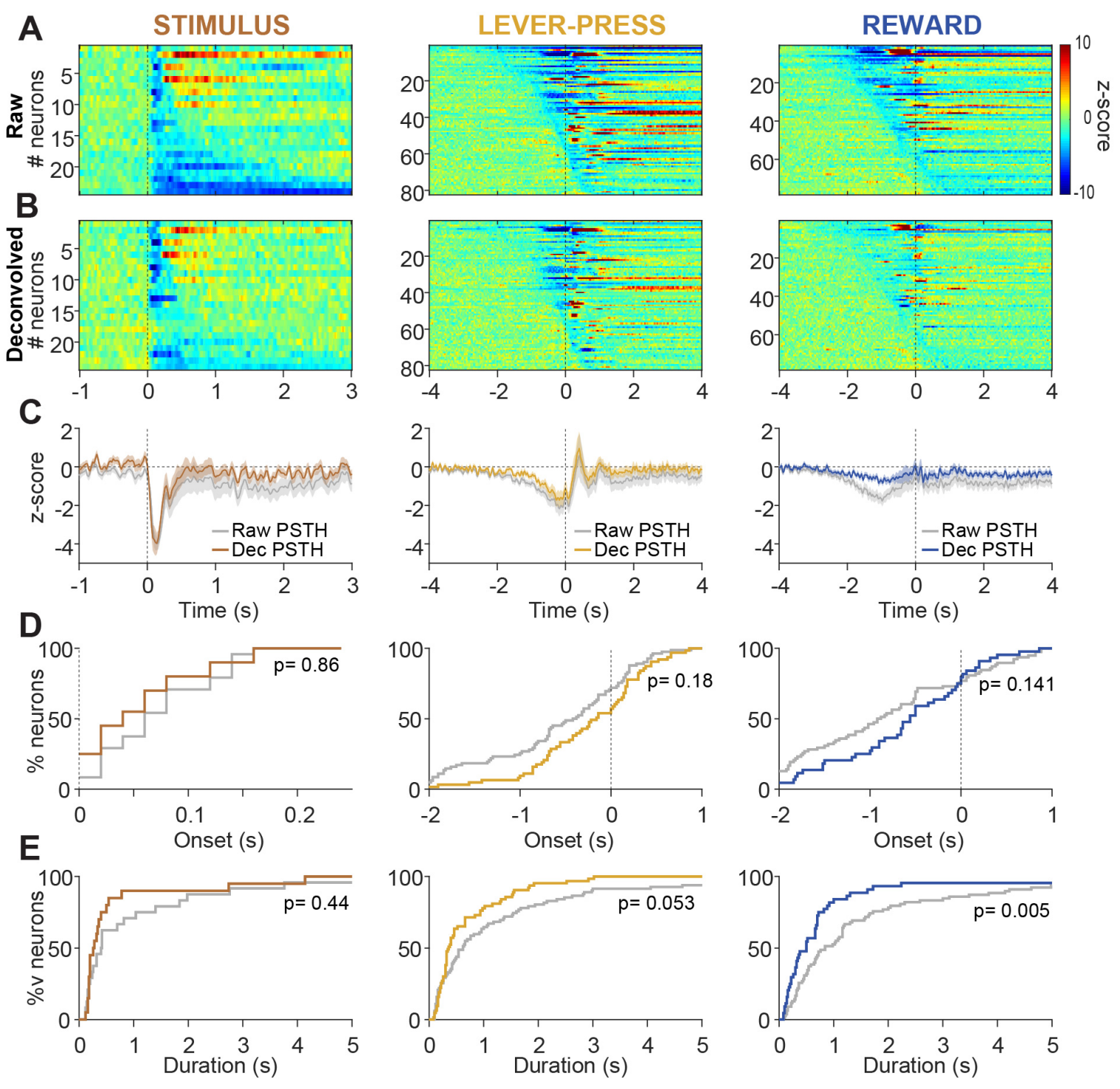

$\mathbf{F}$
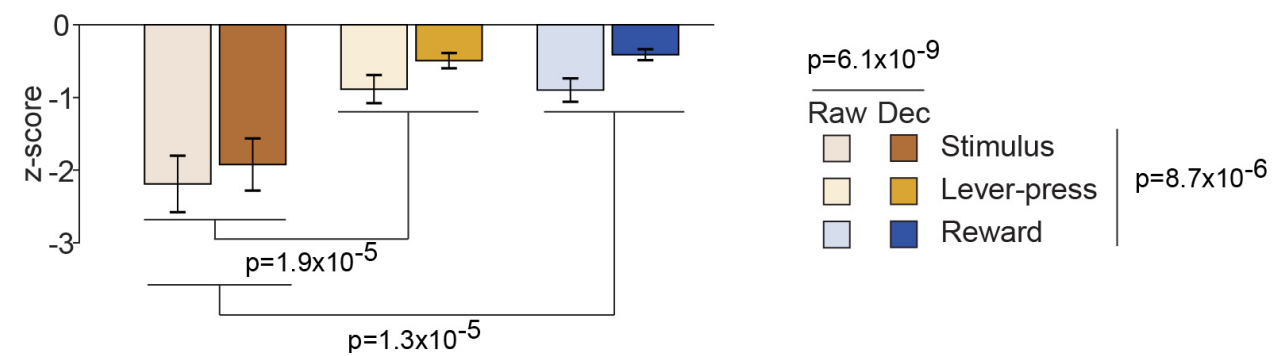

Fig.5. Pf neuronal inhibitions to task events.

A. Heat-maps represent color-coded PSTHs showing neurons inhibited to the stimulus (left), leverpress (middle) and reward delivery (right). Each row represents the PSTH an individual neuron aligned to the event considered. Data are plotted with smoothed $20 \mathrm{~ms}$-time-bins and neurons are sorted by inhibition durations for stimuli responses and onset latencies for lever-press and reward responses. B. Heat-maps represent deconvolved PSTH of the same neurons represented in A. C. Average responses for the neurons shown in A and B. The gray traces correspond to raw PSTHs. Brown, orange and blue traces correspond to the deconvolved PSTHs to the stimulus, lever-press and reward delivery, respectively. D. Cumulative percentage of inhibition onset latencies for raw and deconvolved responses. E. Cumulative percentage of inhibition durations. F. Average z-scores of inhibitions. 


\section{Modulation of stimulus-evoked responses by the motivational state.}

315 Our data showed that Pf neurons are strongly activated by stimuli when the rats engage in reward-seeking. We then sought to decipher whether stimuli-evoked neuronal modulations

317 depended on the motivational state. We took advantage of the fact that long sessions produce 318 enough trials in which the rats engaged in reward-seeking in response to the stimulus 319 (attended trials) and others in which they did not (unattended trials, Fig. 6). Visual inspection 320 of the data revealed very brisk excitations leading us to use a higher time resolution ( $2 \mathrm{~ms}$ ) to 321 construct PSTHs and a shorter response duration requirement to detect excitations (4 ms). 322 Because of the short latency of these responses that most certainly preceded locomotor onset 323 to the stimulus (McGinty et al., 2013), we analyzed neuronal activity on raw and not 324 deconvolved PSTHs. We observed a first population of 62 neurons (15.5\%) with higher phasic activations in response to attended than unattended stimuli (paired t-test, $t_{61}=7.09$, $p=1.63 \times 10^{-9}$, Fig. $\left.6 \mathrm{~A}, \mathrm{~B}\right)$. We named these neurons MOTIV + because their activation to the

327 stimulus reflects the animal's motivation to engage in the task. We also found a second 328 population of 40 neurons (10\%) that displayed an opposite pattern: they were more 329 intensively excited to unattended than attended stimuli (MOTIV- neurons, paired t-test, $\mathrm{t}_{39}=-$ $3304.89, \mathrm{P}=1.77 \times 10^{-5}$, Fig. $\left.6 \mathrm{~A}, \mathrm{~B}\right)$. The remaining neurons did not respond differently to the stimuli 331 on attended and unattended trials.

332 The analysis of the response profile dynamics revealed that MOTIV-neurons were excited at 333 a considerably shorter onset latency than MOTIV+ neurons (30.4+/-3.6 ms and 44.2+/-2.8 ms, 334 respectively, $\mathrm{KS}=0.394, \mathrm{P}=9.81 \times 10^{-4}$, Fig. $6 \mathrm{C}$ ). The excitations of MOTIV- neurons to 335 unattended stimuli were also considerably shorter than those of MOTIV+ neurons to attended 336 stimuli $\left(117+/-29 \mathrm{~ms}\right.$ and $436+/-87 \mathrm{~ms}$, respectively, $\mathrm{KS}=0.403, \mathrm{P}=6.92 \times 10^{-4}$, Fig. $\left.6 \mathrm{C}\right)$.

337 We conducted a similar analysis on stimuli-inhibited neurons on 20 ms-time-based PSTHs (Fig. 338 7) and found 21 MOTIV+ and 3 MOTIV- neurons. MOTIV+ neurons were more inhibited to 339 attended than unattended stimuli (paired $t$-test, $t_{20}=-5.718, p=1.35 \times 10^{-5}$ ). The onset latency 340 to attended stimuli was $78+/-11 \mathrm{~ms}$ and lasted $535+/-132 \mathrm{~ms}$. Given the low number of 341 MOTIV- neurons, we did not conduct any comparison. 

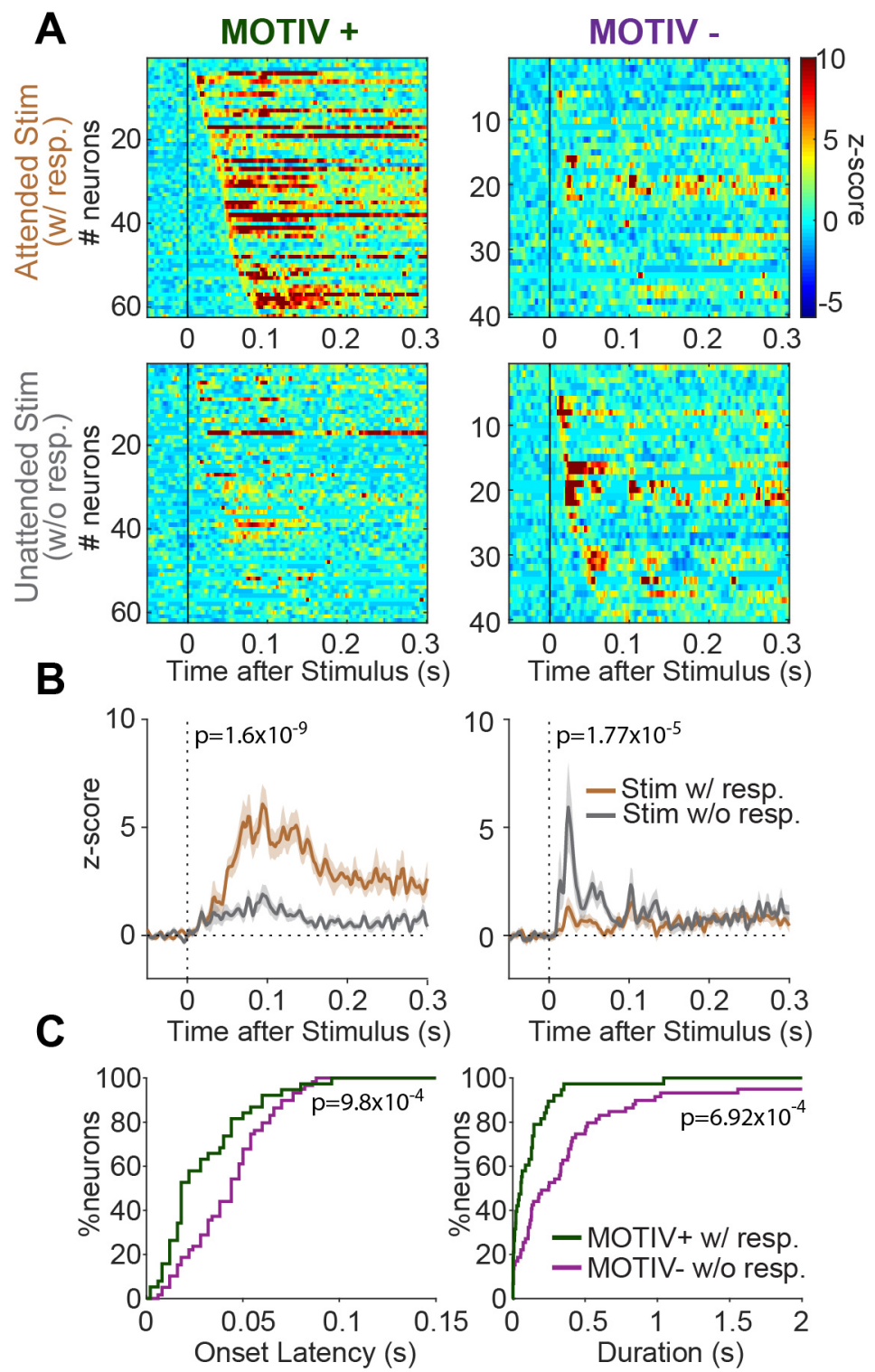

Fig.6. Pf neuronal excitations to the stimulus depend on whether the animal engages in rewardseeking.

A. Heatmaps showing stimuli-evoked excitations of neurons on trials the animals engaged in reward-seeking (top) and those they did not (bottom) for MOTIV+ (left) and MOTIV-(right) neurons. Neurons are sorted by onset latencies. Raw PSTHs are plotted with 2 ms-time-bins. B. Average PSTHs for MOTIV + and MOTIV- neurons for attended (brown) and unattended (gray) stimuli. C. Cumulative percentage of excitation latencies (left) and durations (right) for MOTIV+ (green) and MOTIV- (purple) neurons. 

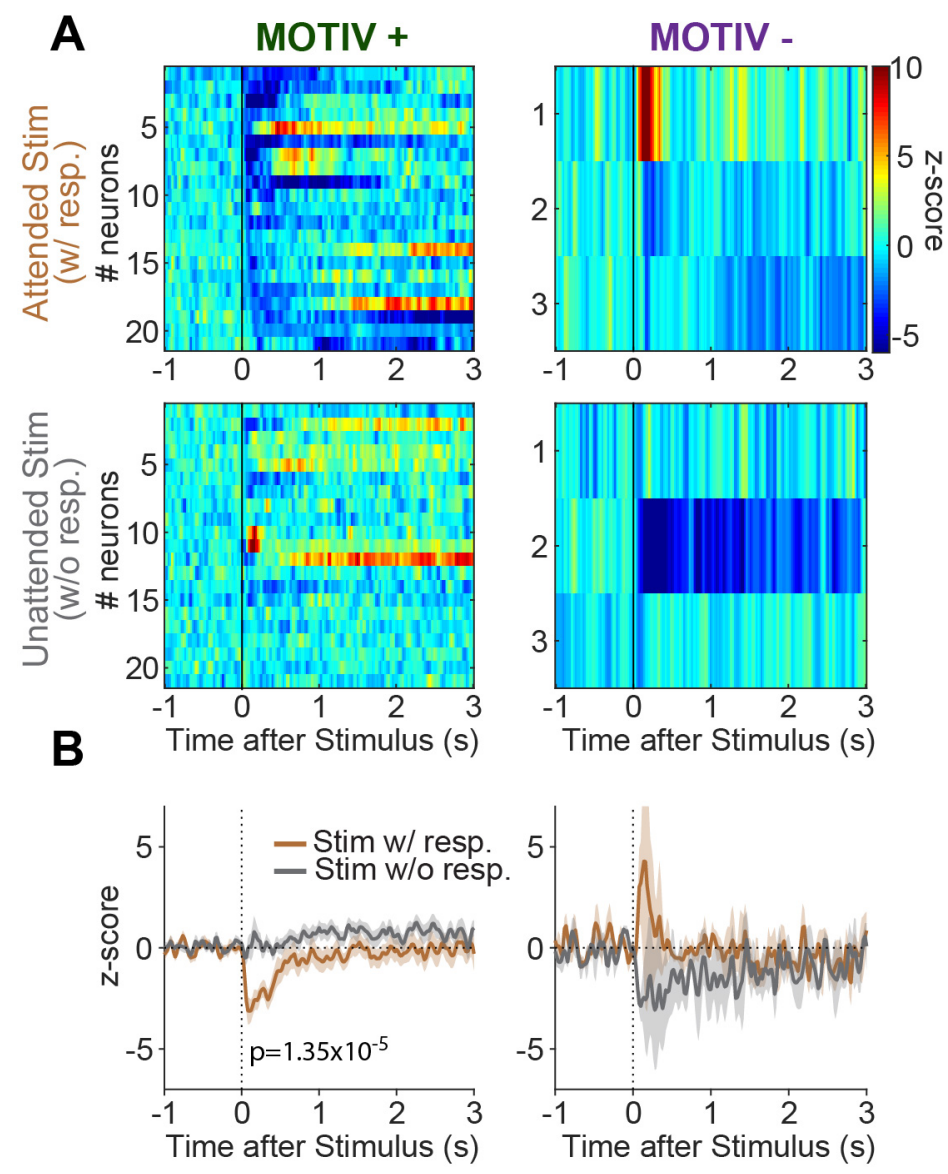

Fig.7. Pf neuronal inhibitions to the stimulus depends on whether the animal engages in reward-seeking.

A. Heatmaps showing stimuli-evoked inhibitions of neurons on trials the animals engaged in reward-seeking (top) and those they did not (bottom) for MOTIV+ (left) and MOTIV- (Iright) neurons. Neurons are sorted by onset latencies. Raw PSTHs are plotted with 20 ms-time-bins. B. Average PSTHs for MOTIV + and MOTIV- neurons for attended (brown) and unattended (gray) stimuli. 


\section{DISCUSSION}

345 We sought to characterize the electrophysiological activity of Pf neurons in rats performing a 346 stimulus-driven reward-seeking task. We show that most Pf neurons respond to the different 347 task events with a higher proportion of excitations than inhibitions. The waveforms and discharge properties were not predictive of their excited or inhibited responses to task events. Stimuli evoked larger responses than the actions of lever-pressing and collecting the rewards. The most striking finding was that excitations to stimuli depended on whether the animal later engaged in reward-seeking.

\section{Basic electrophysiological properties of Pf neurons}

353 Pf neurons have traditionally been identified as a homogeneous population of long-range 354 projection glutamatergic neurons (Wilson et al., 1983; Mouroux and Féger, 1993; Smith et al., 2004). However, several studies have now revealed Pf subtypes with distinct morphological and electrophysiological signatures. Neurons with bushy dendritic trees or diffuse minimally branching dendrites show brief or long after-hyperpolarization, respectively leading to different abilities to sustain high frequency firing (Beatty et al., 2009; Mendez-Rodriguez et al., 2021). Furthermore, a recent study found that the mouse caudal Pf contains a small but significant proportions of GABA neurons intermingled with glutamate neurons (Chen et al., 2020). In vitro, GABA neurons displayed short action potential durations and higher discharge rates induced by current injections than glutamate neurons. To our knowledge, no studies have reported the presence of GABA neurons in rats. In light of these heterogeneous Pf neuron subtypes, we analyzed the basic electrophysiological properties of the neurons recorded extracellularly during the baseline period, when no task events were presented. The

366 firing characteristics were assessed by measuring the basal firing rate and the maximal 367 frequency reached as an index of their bursting abilities. Indeed, bushy neurons were reported to have higher maximal discharge rates and burstiness because of their shorter afterhyperpolarization (Beatty et al., 2009; Mendez-Rodriguez et al., 2021). We did find that all the parameters studied were dispersed but when plotted against each other, they strongly

371 overlapped and we could not determine criteria to cluster different populations.

372 Because we found neurons excited and inhibited to different task events, we sought to 373 determine whether these profiles mapped with their basic electrophysiological properties. 
374 We did find that inhibited neurons had significantly higher basal firing rates and shorter spike 375 widths. But here too, these differences were not sufficient to identify excited or inhibited 376 neurons based on their intrinsic basal firing properties.

\section{Pf neurons are activated during the behavioral approach}

378 We found a very high prevalence of neurons modulated by task events with a strong bias toward excitations. Stimuli clearly evoked the strongest responses, for both excited and inhibited neurons. This is certainly related to the temporal unpredictability of stimuli presentations and their association with rewards that have both been shown to potentiate the magnitude of excitations in the primate CM/Pf (Matsumoto et al., 2001; Minamimoto and Kimura, 2002). We found that stimuli-evoked responses occurred at a relatively constant latency with large variations in their duration. In many cases, we observed that excitations persisted until the rat lever-pressed. The long sessions introduced a large variability in the response latency within and between sessions that likely explained the variability in the durations of excitations. Deconvolution allows to isolate neuronal activity time-locked to the behavioral event considered by removing the contribution of neighboring events (Ghazizadeh et al., 2010; Ambroggi et al., 2011). The fact that deconvolution shortened stimulus- and action-evoked excitations suggests that the behavioral approach by itself activated Pf neurons. Interestingly, such pattern of anticipatory activity seems to be found in both primates and mice (Minamimoto et al., 2014; Díaz-Hernández et al., 2018). In a task where monkeys had to perform specific actions in response to instructive stimuli, the excitation of the classical long-latency facilitation (LLF) neurons recorded in CM appeared more strongly correlated with the timing of the required action than the instructive stimulus directing it 396 (Minamimoto et al., 2014). Furthermore, a local inactivation of the CM with muscimol decreased the number of licks in a pavlovian task (Matsumoto et al., 2001), confirming the contribution of LLF activity on behavioral responding. In mice running a fixed-ratio 8 schedule task, where no explicit stimuli were presented, Pf neurons also displayed an excitation that preceded the first lever-press and that was shown to be necessary. Indeed, an optogenetic inhibition of striatum-projecting Pf neurons increased the latency to re-engage in a trial 402 without affecting the repeated motor sequence of lever-pressing (Díaz-Hernández et al., 403 2018). Altogether, these results indicate that the excitation of Pf neurons during the behavioral approach is causal. 


\section{Influence of the motivational state on Pf responses to incentive stimuli}

406 The use of long sessions allowed to analyze Pf neuronal activity in response to stimuli predicting the same rewards while rats were in different motivational states. We compared the trials to which the animal attended to the stimulus by engaging in the behavioral response (and thus obtaining the reward) and those they did not. The absence of engagement on unattended trials was unlikely due to a failure in perceiving the stimulus that was highly salient ( $85 \mathrm{~dB}$ white noise coupled to the visual extension of the lever) and long lasting (up to $10 \mathrm{~s}$ ). The strongest evidence against this hypothesis is the fact that we identified a population of neurons that was more excited on unattended trials, indicative of an active process taking place in these situations. Most likely, the absence of responding was caused by the fact that at certain times, animals in these long sessions valued reward-seeking less than other activities (e.g. grooming, exploring, resting).

417 MOTIV+ neurons exhibited either excitations or inhibitions to attended stimuli in the first $418100 \mathrm{~ms}$ of their presentations. In a similar task, locomotion onset has been reported to start $\sim 250 \mathrm{~ms}$ after a reward-predictive stimulus, indicating that the initial component of the responses of MOTIV+ neurons was not driven by movements themselves (McGinty et al., 2013) but could participate in the initiation process. It seems unlikely that MOTIV+ neurons are homolog to primate LLF neurons for two reasons. First, LLF neurons usually have a biphasic response with an inhibition preceding the excitation (Matsumoto et al., 2001) which we did not observe in MOTIV+ neurons. Second, the amplitude of the excitatory component of LLF neurons is inversely correlated with the reaction time on a trial-to-trial basis (Minamimoto et al., 2014). The stronger response to attended stimuli compared to unattended stimuli is diametrically opposed to this observation. Thus, the MOTIV+ profile reported in this study does not seem to match LLF activity or any other reported in primates that we are aware of. MOTIV- neurons had a stereotyped response with excitations starting as early as $6 \mathrm{~ms}$ after 430 stimulus onset and lasting less than $100 \mathrm{~ms}$. Yet, these brisk responses were associated with 431 the absence of behavioral responding in the next $10 \mathrm{~s}$. These data provide further evidence 432 that the Pf carries an important attentional function (Minamimoto and Kimura, 2002; 433 Minamimoto et al., 2009; Redgrave et al., 2011; Smith et al., 2011) by gathering low level 434 sensory information that may arise from the deep layers of the superior colliculi and/or the pedunculopontine nucleus (Paré et al., 1988; Krauthamer et al., 1992; Krout et al., 2001). 
436 MOTIV- neurons shared properties with short-latency facilitation neurons (SLF) recorded in 437 the primate Pf (Matsumoto et al., 2001). But importantly, the early and transient firing of 438 MOTIV- neurons manifested when the rat did not attend to stimuli indicates that their attention was not directed toward them. To the contrary, Pf recordings and inactivations in monkeys during a countermanding task provided evidence that SLF neurons participate in the direction of attention toward stimuli (Minamimoto and Kimura, 2002). This apparent 442 discrepancy could relate to the modality used in these studies (auditory and visual in our study 443 and purely visual in the primate study) or even different functions carried by Pf neurons in 444 different species. Another intriguing possibility lies in the characteristics of the stimuli used. 445 Minamimoto and Kimura (2002) presented temporally predictable stimuli that provided 446 instructions to the monkeys about the direction of the saccade to be rewarded. In our study, 447 we used stimuli that were presented unexpectedly and incentivized the rats to switch from their current activity to reward-seeking by engaging in actions during a 10 s-time window.

449 These actions strongly differed depending on the location of the rats at the time of occurrence 450 of these stimuli. Thus, the Pf could subserve different roles for instructive and incentive stimuli 451 as we previously showed for NAc neurons (Sicre et al., 2020).

452 The excitations of MOTIV- neurons could participate in the process of not engaging in actions 453 in response to the incentive stimulus through the dense Pf projection to the striatal complex 454 (Van der Werf et al., 2002). As opposed to other thalamic nuclei, the Pf preferentially synapses on cholinergic interneurons (CINs) (Lapper and Bolam, 1992; Sidibé and Smith, 1999; Raju et 456 al., 2006; Doig et al., 2014) that exert a strong inhibitory control on the activity of medium spiny projection neurons (MSNs) in vivo (Witten et al., 2010) through different GABA interneuron subtypes (English et al., 2012; Assous et al., 2017; Assous and Tepper, 2018). We recently reported that NAc Core CINs were also more active in response to unattended than attended incentive stimuli (Sicre et al., 2020). The short latencies observed in CINs suggest that they could be driven by MOTIV- Pf neurons.

462 Excitations of NAc Core MSNs evoked by incentive stimuli are necessary for rats to engage in 463 action (Ambroggi et al., 2011) and depend on ventral tegmental area (Yun et al., 2004), 464 basolateral amygdala (Ambroggi et al., 2008) and paraventricular (Meffre et al., 2019) inputs. 465 Together, our recent work suggests a circuit able to repress the activation of NAc Core MSNs to incentive stimuli by these inputs when the animal is not willing to engage in action through 
467 the activation of CINs driven by Pf MOTIV- neurons. This circuit may allow to dynamically filter 468 which predictive information is able to control NAc Core MSNs and thus suggest an attentional 469 process directed toward the motivational system.

\section{CONTRIBUTIONS}

471 MS conducted experiments, analyzed data, prepared figures and wrote the initial draft of the 472 manuscript. JM supervised MS, participated to experiments and provided critical comments 473 to the manuscript. FA designed research, analyzed data, prepared figures and edited the 474 manuscript.

\section{FUNDING}

476 This work was supported by the Centre National de la Recherche Scientifique (CNRS) and Aix477 Marseille Université (AMU).

478

\section{ACKNOWLEDGEMENTS}

The authors would like to thank Bruno Poucet for his support, Didier Louber and Dany Paleressompoulle for technical assistance and Simon Moré for information technology assistance.

\section{DECLARATION OF INTEREST}

The authors declare no conflict of interest.

\section{REFERENCES}

Alexander G (1986) Parallel Organization of Functionally Segregated Circuits Linking Basal Ganglia and Cortex. Annu Rev Neurosci 9:357-381

Ambroggi F, Ghazizadeh A, Nicola SM, Fields HL (2011) Roles of nucleus accumbens core and shell in incentive-cue responding and behavioral inhibition. J Neurosci 31:6820-6830.

Ambroggi F, Ishikawa A, Fields HL, Nicola SM (2008) Basolateral Amygdala Neurons Facilitate 

of Vertebrates, Including Man (Macmillan, ed). New York, NY. thalamic information via distinct striatal interneuron circuits. Nat Commun 8:15860

Assous M, Tepper JM (2018) Excitatory extrinsic afferents to striatal interneurons and interactions with striatal microcircuitry. Eur J Neurosci

Beatty JA, Sylwestrak EL, Cox CL (2009) Two distinct populations of projection neurons in the rat lateral parafascicular thalamic nucleus and their cholinergic responsiveness. Neuroscience 162:155-173

Berendse HW, Groenewegen HJ (1990) Organization of the thalamostriatal projections in the 501

Berendse HW, Groenewegen HJ (1991) Restricted cortical termination fields of the midline 503 and Cholinergic Control of Goal-Directed Action: Interlacing New with Existing Learning

Butler AB (1994) The evolution of the dorsal thalamus of jawed vertebrates, including mammals: Cladistic analysis and a new hypothesis. Brain Res Rev 19:29-65.

Díaz-Hernández E, Contreras-López R, Sánchez-Fuentes A, Rodríguez-Sibrían L, RamírezJarquín JO, Tecuapetla F (2018) The Thalamostriatal Projections Contribute to the Initiation and Execution of a Sequence of Movements. Neuron 100:739-752.e5 

94:388-400.e4.

Doig NM, Magill PJ, Apicella P, Bolam JP, Sharott A (2014) Cortical and thalamic excitation mediate the multiphasic responses of striatal cholinergic interneurons to motivationally salient stimuli. J Neurosci 34:3101-3117

du Hoffmann J, Kim JJ, Nicola SM (2011) An inexpensive drivable cannulated microelectrode array for simultaneous unit recording and drug infusion in the same brain nucleus of

English DF, Ibanez-Sandoval O, Stark E, Tecuapetla F, Buzsáki G, Deisseroth K, Tepper JM, Koos T (2012) GABAergic circuits mediate the reinforcement-related signals of striatal cholinergic interneurons. Nat Neurosci 15:123-130

Ghazizadeh A, Fields HL, Ambroggi F (2010) Isolating event-related neuronal responses by deconvolution. J Neurophysiol 104:1790-1802.

Groenewegen HJ, Berendse HW (1994) The specificity of the "nonspecific" midline and intralaminar thalamic nuclei. Trends Neurosci 17:52-57.

Hamlin AS, Clemens KJ, Choi E a, McNally GP (2009) Paraventricular thalamus mediates context-induced reinstatement (renewal) of extinguished reward seeking. Eur J Neurosci

Hikosaka O, Kim HF, Yasuda M, Yamamoto S (2014) Basal Ganglia Circuits for Reward ValueGuided Behavior. Annu Rev Neurosci 37:289-306 3924 [Accessed January 2, 2019]. thalamus, is critical to regulating cue-induced reinstatement of cocaine-seeking. Int J 
Jones EG, Leavitt RY (1974) Retrograde axonal transport and the demonstration of nonspecific projections to the cerebral cortex and striatum from thalamic intralaminar nuclei in the rat, cat and monkey. J Comp Neurol 154:349-377.

Kimura M, Minamimoto T, Matsumoto N, Hori Y (2004) Monitoring and switching of corticobasal ganglia loop functions by the thalamo-striatal system. Neurosci Res 48:355-360

Krauthamer GM, Krol JG, Grunwerg BS (1992) Effect of superior colliculus lesions on sensory unit responses in the intralaminar thalamus of the rat. Brain Res 576:277-286

Krout KE, Loewy a D, Westby GW, Redgrave P (2001) Superior colliculus projections to midline and intralaminar thalamic nuclei of the rat. J Comp Neurol 431:198-216

Lapper SR, Bolam JP (1992) Input from the frontal cortex and the parafascicular nucleus to cholinergic interneurons in the dorsal striatum of the rat. Neuroscience 51:533-545.

Mandelbaum G, Taranda J, Haynes TM, Hochbaum DR, Huang KW, Hyun M, Umadevi

Matsumoto N, Minamimoto T, Graybiel AM, Kimura M (2001) Neurons in the thalamic CM-Pf complex supply striatal neurons with information about behaviorally significant sensory events. J Neurophysiol 85:960-976

McGinty VB, Lardeux S, Taha SA, Kim JJ, Nicola SM (2013) Invigoration of reward seeking by cue and proximity encoding in the nucleus accumbens. Neuron 78:910-922 basal ganglia. Trends Neurosci 28:401-407.

561 Meffre J, Sicre M, Diarra M, Marchessaux F, Paleressompoulle D, Ambroggi F (2019) Orexin in 

perpetuity. It is made available under aCC-BY-NC-ND 4.0 International license. the Posterior Paraventricular Thalamus Mediates Hunger-Related Signals in the Nucleus Accumbens Core. Curr Biol 29:3298-3306.e4

Mendez-Rodriguez BS, Arias-Garcia MA, Tapia D, Laville A, Bargas J, Galarraga E (2021) Firing Differences Between Adult Intralaminar Thalamo-striatal Neurons. Neuroscience

Minamimoto T, Hori Y, Kimura M (2005) Neuroscience: Complementary process to response bias in the centromedian nucleus of the thalamus. Science (80- ) 308:1798-1801.

Minamimoto T, Hori Y, Kimura M (2009) Roles of the thalamic CM-PF complex-Basal ganglia circuit in externally driven rebias of action. Brain Res Bull 78:75-79.

Minamimoto T, Hori Y, Yamanaka K, Kimura M (2014) Neural signal for counteracting preaction bias in the centromedian thalamic nucleus. Front Syst Neurosci 8

Minamimoto T, Kimura M (2002) Participation of the thalamic CM-Pf complex in attentional orienting. J Neurophysiol 87:3090-3101

Mouroux M, Féger J (1993) Evidence that the parafascicular projection to the subthalamic nucleus is glutamatergic. Neuroreport 4:613-615

Paré D, Smith Y, Parent A, Steriade M (1988) Projections of brainstem core cholinergic and non-cholinergic neurons of cat to intralaminar and reticular thalamic nuclei. Neuroscience 25:69-86.

Raju D V, Shah DJ, Wright TM, Hall R a, Smith Y (2006) Differential synaptology of vGluT2containing thalamostriatal afferents between the patch and matrix compartments in rats. J Comp Neurol 499:231-243

Redgrave P, Gurney K (2006) The short-latency dopamine signal: a role in discovering novel actions? Nat Rev Neurosci 7:967-975

Redgrave P, Prescott TJ, Gurney K (1999) The basal ganglia: A vertebrate solution to the selection problem? Neuroscience 89:1009-1023. 
Redgrave P, Vautrelle N, Reynolds JNJ (2011) Functional properties of the basal ganglia's reentrant loop architecture: selection and reinforcement. Neuroscience 198:138-151

Rikhye R V, Wimmer RD, Halassa MM (2018) Toward an Integrative Theory of Thalamic Function. Annu Rev Neurosci 41:163-183

Sicre M, Meffre J, Louber D, Ambroggi F (2020) The nucleus accumbens core is necessary for responding to incentive but not instructive stimuli. J Neurosci 40:1332-1343.

Sidibé M, Smith Y (1999) Thalamic inputs to striatal interneurons in monkeys: Synaptic organization and co-localization of calcium binding proteins. Neuroscience 89:11891208.

Sirigu A, Duhamel JR (2016) Reward and decision processes in the brains of humans and nonhuman primates. Dialogues Clin Neurosci 18:45-53

Smith Y, Raju D V, Pare J-F, Sidibe M (2004) The thalamostriatal system: a highly specific network of the basal ganglia circuitry. Trends Neurosci 27:520-527

Smith Y, Surmeier DJ, Redgrave P, Kimura M (2011) Thalamic Contributions to Basal GangliaRelated Behavioral Switching and Reinforcement. J Neurosci 31:16102-16106

Van der Werf YD, Witter MP, Groenewegen HJ (2002) The intralaminar and midline nuclei of the thalamus. Anatomical and functional evidence for participation in processes of arousal and awareness.

Wilson CJ, Chang HT, Kitai ST (1983) Origins of post synaptic potentials evoked in spiny neostriatal projection neurons by thalamic stimulation in the rat. Exp Brain Res 51:217226.

Witten IB, Lin SC, Brodsky M, Prakash R, Diester I, Anikeeva P, Gradinaru V, Ramakrishnan C, Deisseroth K (2010) Cholinergic interneurons control local circuit activity and cocaine conditioning. Science (80- ) 330:1677-1681. 
bioRxiv preprint doi: https://doi.org/10.1101/2021.02.14.431166; this version posted February $14,2021$. The copyright holder for this preprint (which was not certified by peer review) is the author/funder, who has granted bioRxiv a license to display the preprint in perpetuity. It is made available under aCC-BY-NC-ND 4.0 International license.

610 Yun IA, Wakabayashi Ken T., Fields HL, Nicola SM (2004) The Ventral Tegmental Area Is

611 Required for the Behavioral and Nucleus Accumbens Neuronal Firing Responses to Incentive Cues. J Neurosci 24:2923-2933 